\title{
Avaliação das concentrações de fibrinogênio plasmático em equinos da raça Mangalarga Marchador: efeito do exercício, do gênero e da idade
}

\author{
Evaluation of plasma fibrinogen concentrations in Mangalarga Marchador horses: effect of exercise, \\ gender and age \\ Rodrigo Nogueira Bastos, Thiago L. P. Marques, Márcia Torres Ramos, Maria Fernanda de Mello \\ Costa
}

\begin{abstract}
Resumo
Muitas variáveis fisiológicas foram estudadas ao longo dos anos visando a investigaçãodos efeitos do exercício sobre o corpo equino. O exercício é conhecido por causar alterações fisiológicas no corpo e muitas vezes, quando excessivo, pode resultar em lesão.O fibrinogênio é uma proteína de fase aguda produzida pelo fígado cujas concentrações podem aumentar rapidamente em decorrência de processo inflamatório. Este estudo avaliou as concentrações de fibrinogênio em equinos Mangalarga Marchador, investigando a possibilidade de que o exercício inicia uma resposta inflamatória como parte do processo de adaptação que leva ao condicionamento físico. Sangue venoso foi coletado de 17 equinos hígidos antes e após o exercício e o fibrinogênio foi aferido por precipitação pelo calor. Foram realizados questionários respondidos pelos proprietários e eram referentes ao manejo sanitário dos equinos participantes do estudo. Foi observada diferença nos valores médios de fibrinogênio plasmático obtido antes e após o exercício. Quando se comparou animais que trabalhavam três dias por semana com o grupo que era exercitado sete dias por semana observou-se diferença nos valores médios de fibrinogênio plasmático apenas após o exercício.Os resultados deste estudo indicam que o exercício causou uma resposta inflamatória leve, resultando em aumento do fibrinogênio após o exercício. Parece ser possível a utilizaçãodo fibrinogênio como biomarcador para a inflamação resultante do treinamento esportivo em equinos saudáveis e monitoramentoda resposta fisiológica dos animais a fim de prevenir o treinamento excessivo.

Palavras-chave: Cavalo; Metabolismo; Exercício; Hiperfibrinogenemia; Marcha
\end{abstract}

Marques TLP, Ramos MT, De Mello Costa, MF. Avaliação das concentrações de fibrinogênio plasmático em equinos da raça Mangalarga Marchador: efeito do exercício, do gênero e da idade. Revista de Saúde. 2016 Jul./Dez.; 07 (2):12-15

\begin{abstract}
Many physiological variables have been tested over the years in an attempt to investigate the effects of exercise on the equine body. Exercise is known to cause physiological changes in the body and often overtraining results in injury to equine athletes. Fibrinogen is an acute phase protein synthesized in the liver and its concentrations can increase rapidly as a result of an inflammatory process. This study evaluated fibrinogen concentrations in Mangalarga Marchador horses to investigate the possibility that exercise initiates an inflammatory response as part of the adaptation process that leads to physical conditioning. Venous blood was collected from 17 healthy horses before and after exercise and fibrinogen was measured by heat precipitation. Questionnaires were distributed and answered by the owners of the horses to acquire information regarding management and health care of the animals participating in the study. A significant difference in fibrinogen concentration before and after exercise was observed, as well as a difference in fibrinogen between horses that exercise tree times a week as compared to those who exercised seven times a week. The results from this study indicate that exercise caused a mild inflammatory response, and that chronic exercise exacerbates this response. It appears to be possible to use fibrinogen concentration as a biomarker for inflammation produced as a result of training in healthy horses and monitor the physiological response of animals with the aim of preventing overtraining.

Keywords: Horse; Metabolism; Exercise; Hyperfibrinogenemia; Marcha
\end{abstract}

\section{Introdução}

A distribuição mundial da população de equinos indica a importância da utilização econômica destes animais, além de expressar aspectos culturais dos países e continentes. As Américas possuem 57,2 \% da população mundial de equinos, tendo o Brasil uma população estimada de 5.541.702 equinos, sendo o quarto maior rebanho do mundo. ${ }^{2}$
Os estudos em fisiologia do exercício através de testes para a avaliação do desempenho atlético realizados a campo (pista), juntamente com as respostas fisiológicas ao exercício e ao treinamento, constituem uma valiosa ferramenta na avaliação atlética dos equinos. ${ }^{3,9}$

O fibrinogênio é uma proteína de fase aguda produzida pelo fígado, constituindo cerca de $5 \%$ das proteínas plasmáticas totais. Em equinos hígidos

\footnotetext{
Afiliação dos autores: Universidade Severino Sombra.

* Endereço para correspondência: Universidade Severino Sombra, Av. Exped. Oswaldo de Almeida Ramos, 280 - Centro - Vassouras,RJ - CEP 27700-000.

E-mail: marciatramos@gmail.com
} 
as concentrações plasmáticas de fibrinogênio podem variar entre 200 e $400 \mathrm{mg}^{\mathrm{dL}} \mathrm{dL}^{-1}$, tendo como limites máximos aceitáveis valores entre 100 e $500 \mathrm{mg} \cdot \mathrm{dL}^{-1} .4,8$ Segundo Campbell (1981), as concentrações podem aumentar rapidamente em decorrência de processo inflamatório em fase aguda, até valores entre 500 e 600 $\mathrm{mg} . \mathrm{dL}^{-1}$, ou ainda alcançar valores iguais ou superiores

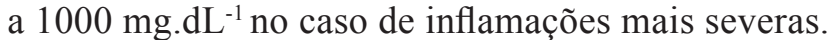

Em humanos já foi demonstrado o aumento transitório do fibrinogênio induzido pelo exercício agudo. ${ }^{6}$ Em equinos, informações divergentes têm sido apresentadas, de maneira que se supõe que as diferenças apresentadas possam ser atribuídas à intensidade e à duração do exercício realizado, aos momentos de coleta e ao método de análise. ${ }^{7}$ Desta forma, ainda é escasso o conhecimento sobre os efeitos do exercício agudo e crônico sobre as concentrações de fibrinogênio plasmático em equinos da raça Mangalarga Marchador.

Este estudo avaliou a influência do exercício, do gênero e da idade do animal sobre as alterações do fibrinogênio plasmático em equinos da raça Mangalarga Marchador

\section{Materiais e métodos}

Foram avaliados 17 equinos hígidos, entre três e 18 anos de idade, da raça Mangalarga Marchador e criados com a finalidade de realizar cavalgadas e competições de marcha. A frequência na qual estes animais realizavam treinamento variou entre um a sete dias por semana.

Foi realizada coleta de $10 \mathrm{~mL}$ de sangue venoso diretamente da veia jugular externa por sistema de vácuo em frasco coletor com EDTA tripotássico, nos momentos imediatamente antes e após uma cavalgada de aproximadamente $10 \mathrm{Km}$. O material coletado foi acondicionado em gelo e remetido para um laboratório privado. Os valores de fibrinogênio foram obtidos pela técnica clássica de precipitação pelo calor. ${ }^{8}$ Também foi realizada entrevista com os proprietários dos equinos, através da qual se obteve informação sobre idade, raça, gênero, manejo alimentar, sanitário, e frequência da prática de atividades físicas.

Os dados foram submetidos às seguintes análises estatísticas: estatística descritiva, teste $\mathrm{T}$ pareado para amostras antes e após o exercício e teste $\mathrm{T}$ para amostras repetidas independentes para investigação da diferença das médias do fibrinogênio entre machos e fêmeas e análise de variância para investigação do efeito da idade e da frequência do exercício.

\section{Resultados}

Dos 17 animais avaliados, dez (58,8 \%) eram machos e sete $(41,2 \%)$ eram fêmeas. Com relação ao resultado dos questionários, a maioria dos animais era vacinada com vacina antirrábica $(70,6$ $\%)$ e mantida a pasto $(76,5 \%)$. Além disso, todos os animais encontravam-se vermifugados. $\mathrm{O}$ treinamento dos animais consistia de exercício realizado um dia semanal $(\mathrm{n}=1 ; 5,9 \%)$, dois $(\mathrm{n}=3 ; 17,6 \%)$, três $(\mathrm{n}=7$; $41,2 \%)$, quatro $(n=1 ; 5,9 \%)$ e sete $(n=5 ; 29,4 \%)$ dias semanais.

Marcantes diferenças individuais foram observadas entre as idades, os valores aferidos das concentrações de fibrinogênio antes e após o exercício, bem como na frequência da realização de atividades físicas semanais, conforme descrito na tabela 1 .

Foi observada diferença significativa nos valores médios de fibrinogênio plasmático entre os momentos antes e após o exercício $(\mathrm{p}=0,004)$ (Tabela 2).

Não foi observada influência do gênero ou

Tabela 1. Descrição dos animais e valores individuais da variável fibrinogênio plasmático obtido antes e após exercício de cavalgada em equinos Mangalarga Marchador.

\begin{tabular}{cccccc}
\hline ANIMLL & SEXO & IDADE & $\begin{array}{c}\text { FIB } \\
\text { ANTES }\end{array}$ & $\begin{array}{c}\text { FIB } \\
\text { APÓ }\end{array}$ & EXERCÍCIO \\
\hline 1 & F & 6 & 200 & 400 & 3 \\
2 & M & 10 & 200 & 200 & 4 \\
3 & M & 7 & 400 & 600 & 7 \\
4 & M & 3 & 200 & 400 & 7 \\
5 & F & 7 & 400 & 600 & 7 \\
6 & M & 5 & 600 & 600 & 7 \\
7 & M & 4 & 600 & 800 & 3 \\
8 & F & 8 & 200 & 200 & 3 \\
9 & M & 4 & 200 & 200 & 2 \\
10 & M & 3 & 200 & 400 & 3 \\
11 & F & 9 & 600 & 600 & 3 \\
12 & F & 7 & 400 & 400 & 3 \\
13 & M & 8 & 400 & 400 & 3 \\
14 & M & 5 & 200 & 400 & 2 \\
15 & F & 7 & 400 & 400 & 2 \\
16 & F & 10 & 200 & 200 & 1 \\
17 & M & 18 & 200 & 200 & 4 \\
Média & - & 7 & 329 & 412 & 2 \\
DP & - & 4 & 157 & 180 & \\
\hline
\end{tabular}

FIB ANTES: fibrinogênio plasmático antes do exercício; FIB DEPOIS: fibrinogênio plasmático após o exercício; EXERCÍCIO: número de dias em que o animal era submetido a exercício em uma semana 
da idade dos animais nos valores médios de fibrinogênio plasmático antes e após o exercício de cavalgada $(\mathrm{p}>0,05)$.

Observou-se diferença significativa nos valores médios de fibrinogênio plasmático após o exercício quando levado em consideração o grupo de animais que realizava treinamento três dias por semana, e o grupo que era exercitado sete dias por

Tabela 2. Valores médios do fibrinogênio plasmático obtidos antes e após o exercício de cavalgada em equinos Mangalarga Marchador

Momento

Fibrinogênio (mg • dL-1)

\begin{tabular}{cc}
\hline Antes & $329 \pm 157^{\mathrm{b}}$ \\
Após & $412 \pm 180^{\mathrm{a}}$ \\
p valor & 0,004
\end{tabular}

Médias nas colunas, seguidas por letras minúsculas diferentes, diferem entre si pelo teste $\mathrm{T}$ pareado $(\mathrm{p}<0,05)$

semana $(p=0,024)$, como visto na Tabela 3.

Discussão e Conclusões

O exercício representa um desafio à fisiologia, levando à modificações em diversos processos químicos e celulares, geralmente dependentes

\begin{tabular}{|c|c|c|c|}
\hline Exercício & 3 & 7 & p valor \\
\hline & Dias/Semanas & Dias/Semanas & \\
\hline $\mathrm{n}$ & 7 & 5 & \\
\hline $\begin{array}{c}\text { Fibrinogênio } \\
\left(\mathrm{mg}^{-} \mathrm{dL}^{-1}\right)\end{array}$ & $372 \pm 138^{b}$ & $600 \pm 141^{a}$ & 0,024 \\
\hline
\end{tabular}

Médias nas linhas, seguidas por letras minúsculas diferentes, diferem entre si pelo teste $\mathrm{T}(\mathrm{p}<0,05)$.

da sua duração e intensidade. ${ }^{5}$ Estudos apontam controvérsias com relação às respostas das concentrações plasmáticas de fibrinogênio induzidas pelo exercício. Piccione e colaboradores (2014) estudando equinos durante treinamento prolongado e de intensidade moderada, observaram a elevação das concentrações de fibrinogênio em resposta ao aumento da carga de exercício. Em contrapartida, Menzies-Gowe e colaboradores avaliando equinos após exercício de baixa intensidade, a passo e trote, não observaram incremento das concentrações plasmáticas de fibrinogênio. Neste estudo observou-se a elevação significativa das concentrações plasmáticas de fibrinogênio após a realização de exercício de cavalgada.

Diversas funções são atribuídas ao fibrinogênio. Entre elas está o fornecimento de substrato para a formação da fibrina do tecido de reparação, assim como provimento de matriz para a migração de células relacionadas à inflamação, iniciando a cascata de sinalização intracelular de inflamação, aumentando a degranulação e os mecanismos de fagocitose. Assim, nas últimas décadas, as concentrações plasmáticas de fibrinogênio têm sido amplamente utilizadas no monitoramento de condições inflamatórias em equinos. ${ }^{1}$ Os resultados deste estudo indicam que o exercício causou uma resposta inflamatória leve, resultando em aumento significativo das concentrações plasmáticas de fibrinogênio após o exercício.

Outrossim, observou-se que nos equinos que eram treinados diariamente as concentrações plasmáticas de fibrinogênio após o exercício eram significativamente mais altas do que aquelas aferidas nos animais treinados somente três dias durante a semana, indicando que o exercício crônico pode exacerbar essa resposta inflamatória.

Desta maneira, parece ser possível a utilização da concentração de fibrinogénio plasmático como biomarcadorpara ainflamaçãoresultante dotreinamento esportivo em equinos saudáveis e monitoramento da resposta fisiológica dos animais com a finalidade de prevenir o treinamento excessivo.

\section{Declarações}

Os autores RNB, TLMP e MTR declaram não possuírem conflitos de interesse diretos ou indiretos. A autora MFMC declara ter vínculo com a Editora 
das Revistas Online da USS, posterior ao envio deste trabalho para publicação.

\section{Comitê de Ética}

Este trabalho foi aprovado pelo CEUA da USS sob o número 042/2016.

\section{Bibliografia}

1. Crisman MV, Scarratt WK, Zimmerman KL. Blood proteins and inflammation in the horse. Veterinary Clinics of North America: Equine Practice. 2008;24(2):285-97.

2. De Almeida FQ, Silva VP. Progresso científico em equideocultura na 1a década do século XXI. Oceania. 2010;411(9.000):420.956.

3. Campbell M, Bellamy J, Searcy G. Determination of plasma fibrinogen concentration in the horse. American journal of veterinary research. $1981 ; 42(1): 100-4$

4. Evans D, Hinchcliff K, Geor R, Kaneps A. Exercise testing in the field. In: Hinchcliff KW KA, Geor RJ, editor. Equine exercise physiology: The science of exercise in the athletic horse. Philadelphia: Elsevier; 2008

5. Fazio F, Assenza A, Tosto F, Casella S, Piccione G, Caola G. Modifications of some acute phase proteins and the white blood cell count in thoroughbreds during training. Veterinary Record: Journal of the British Veterinary Association. 2010;167(9)

6. Kaneko JJ, Harvey JW, Bruss ML. Clinical biochemistry of domestic animals: Academic press; 2008.

7. Menzies-Gow N, Wray H, Bailey S, Harris P, Elliott J. The effect of exercise on plasma concentrations of inflammatory markers in normal and previously laminitic ponies. Equine veterinary journal. 2014;46(3):317-21.

8. Montgomery HE, Clarkson P, Nwose O, Mikailidis D, Jagroop I, Dollery $\mathrm{C}$, et al. The acute rise in plasma fibrinogen concentration with exercise is influenced by the G-453-A polymorphism of the $\beta$-fibrinogen gene. Arteriosclerosis, thrombosis, and vascular biology. 1996;16(3):386-91.

9. Piccione G, Bazzano M, Giannetto C, Marafioti S, Fazio F. Traininginduced changes in clotting parameters of athletic horses. Journal of veterinary science. 2014;15(1):45-9.

10. Schalm OW, Jain NC, Carroll EJ. Veterinary hematology: Lea \& Febiger.; 1975 .

11. Van Erck E, Votion D-M, Serteyn D, Art T. Evaluation of oxygen consumption during field exercise tests in Standardbred trotters. Equine and Comparative Exercise Physiology. 2007;4(01):43-9. 